\title{
El programa de Lamarck: una visión materialista de la vida*
}

Lamarck's Program: A Materialist View of Life

\author{
Ricardo Noguera Solano ${ }^{\dagger}$ \\ Juan Manuel Rodríguez Caso ${ }^{\ddagger}$ \\ Rodrigo Bustillo Ramirez ${ }^{\S}$
}

\begin{abstract}
Resumen
En este escrito se argumenta que la propuesta de Lamarck sobre la transformación está construida a partir de un enfoque materialista, tanto en sentido filosófico como metodológico. El primero entendido como una práctica científica, una de las tendencias del siglo XIX herederas del mecanicismo cartesiano, y el segundo como parte de una corriente del materialismo filosófico francés que reivindicaba la primacía de la materia sobre la conciencia. El primer tipo de materialismo es claramente visible a través de la práctica científica de Lamarck, afianzada en la idea de la naturaleza como causa de los fenómenos naturales y la metodología científica como un instrumento del conocimiento de esas causas. Sobre el segundo tipo de materialismo podemos encontrar algunos destellos discursivos en algunas de sus obras, relacionadas con la idea de "la eternidad de la materia" y la de la "inmortalidad del alma", así como la idea de "variación infinita de las formas orgánicas", y el origen natural e histórico de lo físico y lo moral en el ser humano.
\end{abstract}

Palabras clave: Lamarck - materialismo filosófico - materialismo metodológico - deísmo

\begin{abstract}
In this paper, we argue that Lamarck's theories of transformism are based on a materialistic approach, in both the philosophical and methodological senses. The former is to be understood as scientific practice, a nineteenth-century tendency, heir to Cartesian mechanism, and the latter as part of a current in French philosophical materialism that asserted the primacy of matter over consciousness. The first type of materialism may be clearly observed in Lamarck's own scientific practice, secured to the idea of nature as the cause of natural phenomena and scientific methodology as an instrument for knowing of these causes. Regarding the second type of materialism, occasional instances may be found in some of his works relating to the idea of the 'eternity of matter' and the 'immortality of the soul', as well as the idea of the 'infinite variation of organic forms' and the natural and historical origin of the physical and the moral in the human being.
\end{abstract}

Keywords: Lamarck - philosophical materialism - methodological materialism - deism

\footnotetext{
${ }^{*}$ Recibido: 30 de Marzo de 2016. Aceptado con revisiones: 5 de Diciembre de 2016.

† Facultad de Ciencias, Universidad Nacional Autónoma de México. Para contactar al autor, por favor escribir a: rns@ciencias.unam.mx.

* Posgrado en Filosofía de la Ciencia, Universidad Nacional Autónoma de México. Para contactar al autor, por favor, escribir a: juan_manuel_rodriguez_caso@yahoo.es.

$\$$ Posgrado en Filosofía de la Ciencia, Universidad Nacional Autónoma de México. Para contactar al autor, por favor, escribir a: rodrigo.academiafc@gmail.com.

Metatheoria 8(2)(2018): 147-156. ISSN 1853-2322.

(c) Editorial de la Universidad Nacional de Tres de Febrero. Publicado en la República Argentina.
} 


\section{Introducción}

No escribo para aquellos que buscan nuevos libros, casi siempre con la intención de encontrar sus puntos de vista defendidos; sino para los pocos que leen, que meditan profundamente, que aman el estudio de la naturaleza, y que son capaces de sacrificar incluso su propio interés, al conocimiento de una nueva verdad (Lamarck 1802, p. 2).

Lo anterior fue escrito por Jean-Baptiste Lamarck en Hidrogeología (Lamarck 1802), obra en la que planteaba que el estudio detallado de los fenómenos físicos y químicos era la única vía para entender la naturaleza de los fenómenos orgánicos.

Después de su obra botánica de 1779, Flora francesa (Lamarck 1779), se centró en un programa filosófico, cimentado en un terreno estrictamente materialista, tanto en el sentido del materialismo metodológico, al considerar que todo se origina a partir de la propia naturaleza:

El alcance y dimensiones de lo material, surge necesariamente de su forma, cuyas variedades producidas por infinitas modificaciones de este material son la admirable belleza del universo y de alguna manera la fertilidad de la naturaleza (Lamarck 1794, p. 20).

como de un materialismo filosófico, sosteniendo que la naturaleza podía ser causa de sí misma: "La cantidad de materia no puede aumentar o disminuir, porque ese poder que podría crear, sólo podría destruirla y ni siquiera es necesario volverla a la nada" (Lamarck 1794, p. 21). Esta visión se utilizará, posteriormente, de manera ambigua tanto en Filosofía zoológica (1809, p. 361), como en Sistema analítico del conocimiento positivo del hombre de 1820, en donde Lamarck utilizará la palabra DIEU (Lamarck 1820, pp. $8,10-12,15,40,41,44,74)$, en un sentido mucho más literal, lo cual contrastaría con una postura mucho más materialista de sus primeras obras. La explicación, desde nuestro punto de vista, no es que Lamarck hubiera abandonado sus posturas materialistas, tanto metodológicas como filosóficas, por posturas deístas, o panteístas, sino que puede explicarse como un acomodo al control de las publicaciones que se dio después de la restauración de la monarquía (Corsi 1987, Corsi 2012) particularmente durante las presiones de censura que ejerció la monarquía napoleónica (Coffin 1917, Polowetzky 1993). Como ya lo señaló León Szyfman, "un análisis profundo de su obra demuestra [que Lamarck] profesaba una doctrina sin ambigüedades" (Szyfman 1982, p. 261), en la que el materialismo está presente en toda su obra, y que como también lo ha señalado Gabriel Gohau, existe una coincidencia en diversos autores sobre caracterizar a Lamarck como un materialista, aunque con diferentes matices: un reduccionista de acuerdo a Bernard Andrieu, un deísta según Goulven Laurent, o como un científico en palabras de François Russo (Gohau en Corsi et al. 2006, p. 10).

En varios sentidos consideramos que Lamarck profeso tanto el materialismo filosófico (ontológico) como el metodológico. Esta división nos permite comprender en otro sentido histórico la propuesta de Lamarck, en la que el hincapié no está únicamente en plantear una metodología concreta para explicar los fenómenos naturales y sus causas, sino entender de manera congruente las consecuencias que se derivan de una explicación netamente materialista.

Dada las consideraciones anteriores, el objetivo de este trabajo es mostrar que las ideas más relevantes de Lamarck que sostienen el marco general de su interpretación de la vida y su búsqueda por construir una filosofía zoológica que le permitiera comprender y explicar la transformación gradual de las especies y el origen de la naturaleza física y moral del ser humano, fueron ideas construidas desde una perspectiva materialista, tanto en el sentido metodológico como en el sentido filosófico.

A partir de lo que Lamarck denomina la tendencia de las formas orgánicas a la perfección diversos autores tanto del pasado como del presente han sostenido que ese elemento "teleológico" es congruente con las ideas de Lamarck sobre la existencia de un Ser superior. Por lo tanto -se sostieneque tiene sentido que en su obra Filosofía zoológica (1809), Lamarck se refiera en algunos párrafos a la idea de un Creador sin ningún conflicto con los principios de la transformación de las especies, asumiendo desde luego que la postura idealista de Lamarck era una postura deísta, un punto destacado por uno de sus biógrafos más conocidos, Alpheus S. Packard (Packard 1901, p. 372). Sin embargo, 
como queremos mostrar, Lamarck tenía ideas materialistas, distintas al deísmo, aunque ambas tendencias estaban presentes en el contexto de la Francia ilustrada, y aunque era común encontrar autores deístas, había autores materialistas en el sentido filosófico, que mantenían una postura similar a la de Jean Meslier (1664-1729) (una biografía breve sobre Meslier puede verse en Onfray 2015).

Meslier, mucho antes que Lamarck había puesto en entredicho la existencia de Dios y la creación divina del ser humano, al argumentar en contra del cartesianismo a partir del materialismo filosófico (Meslier 1729). Meslier, como explicaremos más adelante, propuso un materialismo filosófico y radical en contra de toda creencia religiosa, ya fuera ésta teísta o deísta, con afirmaciones como "la materia ha existido por toda la eternidad" y "no es imposible concebir que [...] la materia sea por si misma lo que es" (Meslier [1729] 1978, p. 123) o la siguiente afirmación que contrasta con una aceptación de creación:

Es evidente que reconociendo a la materia sola como primera causa, como el ser eterno e independiente, se evitaría con ello muchas dificultades insuperables, que se encuentran necesariamente en el sistema de la creación, y con ello se explicaría bastante fácilmente la formación de todas las cosas (Meslier [1729] 1978, p. 124).

Una consecuencia directa de sus afirmaciones era su aceptación de que las creencias de las religiones eran falsas (como la idea de la existencia de Dios y la idea de la inmortalidad del alma), y consideraba que sobre la base de esas creencias se habían construido, relaciones y estructuras políticas, sociales, económicas y religiosas, así como una serie de valores morales que era necesario transformar. Otra consecuencia de su materialismo consiste en reconocer que todas las cosas de la naturaleza, incluidas la plantas, los animales y el ser humano, "son producciones de la naturaleza que surgen de movimientos regulares e irregulares de la materia” (Meslier [1729] 1978, pp. 98-105).

Meslier decía también que:

Así pues, todas las obras y todas las producciones de la naturaleza se hacen necesariamente por causas necesarias y fortuitas, y por causas ciegas y enteramente privadas de razón, y de este modo ni estás obras ni estas producciones demuestran y prueban la existencia de un Dios que las haya formado como vemos (Meslier [1729] 1978, p. 105).

Para diferenciar y contrastar el materialismo filosófico de Meslier consideramos importante retomar la caracterización que Guy Chaussinand-Nogaret hace de deísmo y teísmo:

Para el deísta, como Voltaire, Dios es una especie de mecánico o relojero de precisión impresionante que creó el mundo y le dio las leyes eternas de la operación en las que ya no tiene que intervenir. Por lo tanto, no hay necesidad de orar y esperar a una intervención. Podemos dar gracias por su creación, pero sería absurdo creer que él puede cambiar el mecanismo que introdujo. Esto obviamente excluye los milagros. El teísta, por el contrario, es una fe viva, no sólo una profesión teórica, sino una religión muy profunda. Es una creencia íntima que implica una relación personal con lo divino (Le Roy Ladurie \& Chaussinand-Nogaret 2010, p. 941).

Como podemos ver, el materialismo de Meslier era una aceptación llana de que la Naturaleza es causa de misma, es eterna y "Dios" es una idea que las fibras del cerebro humano han creado. Por su parte una postura deísta asume que Dios existe, ha creado las leyes naturales, pero hay una independencia entre Dios y la naturaleza, entre Dios y el ser humano. Por último, bajo una postura teísta se asume que Dios ha creado la naturaleza, las leyes naturales, y mantiene una relación directa con la naturaleza y con el destino del ser humano.

Ahora bien, el asumir alguna de esas posturas es algo distinto e independiente si un autor es materialista en el sentido metodológico, que significaría realizar investigaciones sobre causas naturales, sin tener necesidad de que en la explicación causal se tenga que explicar cuál es la causa última del origen de dichas causas.

Para mostrar las conexiones del materialismo tanto metodológico como filosófico en las ideas de Lamarck desarrollamos tres puntos, que aun cuando parecieran ser congruentes con alguna postura deísta (o en algunos párrafos, teísta), tienen mayor sentido bajo una visión materialista de la vida. Estos puntos son: i) la explicación de los fenómenos naturales debía darse exclusivamente en términos 
materiales. ii) la idea de "especie" como un concepto arbitrario planteada a partir de la idea de "variación infinita" y de la idea de la diferenciación continua de la descendencia que ocurre bajo una variación infinita de las circunstancias. iii) lo físico y lo moral en el ser humano como resultado de causas naturales. De hecho, como lo ha señalado Jacques Roger, Lamarck fue un materialista al no considerar necesario explicaciones de tipo espiritual, y aunque, como ya lo mencionamos, se le puede caracterizar como un deísta, su postura sobre esto fue vaga, en la medida que buscaba explicaciones materiales para los fenómenos naturales (Roger en Lindberg \& Numbers 1986, p. 291).

\section{Materialismo metodológico: el origen de la vida}

Lamarck publicó en 1809 la primera argumentación coherente sobre la transformación de las especies en su Filosofía zoológica. Entre otras cosas, en esta obra incluyó explicaciones que dan cuenta del origen de nuevas especies. El origen de esas ideas transformistas puede ubicarse desde 1802, en materiales que había escrito para sus clases, y en los que refleja una preocupación por la integración de una ciencia (la biología) que abarcara el estudio de todas las formas vivas, bajo un conjunto de principios filosóficos relativo a lo vivo que estuvieran basados exclusivamente en causas naturales. En esa obra desarrolló toda una argumentación consistente para sostener que la teoría sobre los cuerpos vivos o la biología debía contar con un conjunto de principios filosóficos que dieran cuenta del hecho más significativo de la vida: su transformación.

Lamarck planteaba que los fenómenos biológicos podían explicarse en términos de causas naturales, al mismo tiempo que consideraba que las características de los seres permitían su clasificación, pero la investigación no podía limitarse exclusivamente a ello, y para cambiar eso, propuso que habría que pasar de describir a los seres a explicar los procesos vitales (vitales en el sentido del conjunto de características de lo vivo, y no en el sentido espiritualista), en este sentido, los naturalistas debían también preocuparse por el origen de los organismos, su anatomía, su fisiología, su comportamiento, las estrategias de reproducción, entre otras.

Este conjunto de ideas era lo que significaba para Lamarck hacer filosofía, es decir, la tarea del naturalista no sólo debía consistir en describir la naturaleza, sino explicarla en términos de sus causas y sus relaciones naturales, y lo más importante poder llevar este conjunto de principios a la explicación de la naturaleza física y moral del ser humano; los principios filosóficos también debían explicar las causas del estado en la organización de los seres tal como se observan y en el caso de los organismos de mayor complejidad, explicar el desarrollo de las facultades que presentan y en el caso particular del ser humano, su conciencia, su intelecto y su moral (Lamarck 1809 (I), p. 3).

Lamarck practicaba un materialismo metodológico, el cual, en muchos autores, no era inconsistente con la aceptación de Dios como responsable del origen de universo y sus leyes (Roger en Lindberg \& Numbers 1986, p. 291, Charbonnat 2010). Lamarck utilizaba la ciencia como un instrumento para explicar los fenómenos naturales basándose en causas naturales, sin embargo, por declaraciones en sus propios textos sobre la existencia de un Ser superior inmaterial, y por considerar que "el creador no podía haber previsto todas las contingencias en las que se encuentra sumergida la dinámica de la vida, dándole a cada especie, una organización constante, así como una forma determinada e invariable en sus partes" (Lamarck 1809 (I), p. 265), se ha considerado que Lamarck mantenía una postura que podemos caracterizar como deísta.

Sabemos que justo antes de la Revolución Francesa creció con fuerza un movimiento materialista filosófico desarrollado por el ya mencionado sacerdote Meslier, y fuertemente impulsado por otros ilustrados, pero desconocemos mucho sobre cómo se fueron tejiendo las ideas materialistas en el sentido filosófico en la primera reflexión extensamente argumentada sobre la transformación de las especies. La postura de Meslier ponía en entredicho la creación divina, cosa que señala en su único trabajo, cuyo título abreviado es Mémoire des pensées et sentiments de Jean Meslier, prêtre-curé d'Etrépigny et de Balaives, y que ha sido divulgado con otros nombres como El Testamento de Jean Meslier. 
Las ideas contenidas en la obra de Meslier se divulgaron en París y en Francia, primero como el Testamento de Jean Meslier a partir de una versión impresa en Holanda y, después, popularizadas en distintos resúmenes. Que, en palabras de Voltaire: "Se han hecho varios pequeños resúmenes. Algunos de los cuales han sido impresos; por fortuna, están purgados del veneno del ateísmo" (citado en Meslier 1729, p. 167). Más tarde, en 1762, Voltaire también publicaría un pequeño extracto en una versión menos radical, entre los círculos ilustrados de París y Francia. Otra versión importante, que presentó extractos mucho más cercanos al original, fue aquella que publicó el Barón de Holbach con el título de El buen sentido del Cura Meslier (Holbach 1822) en 1791.

Voltaire, como seguramente otros autores, también hizo una distribución de las ideas de Meslier a través de su correspondencia. Entre los autores a los que les escribió y, que además les envió el libro, se encontraron: Jean le Rond d'Alembert, Conde D'Argental, Étienne Noël Damilaville, su sobrina madame De Florian, Marques D'Argens y Claude Adrien Helvetius. La popularidad de Meslier se puede ver reflejada en la distribución de su obra como lo muestra la carta de Voltaire a Damilaville del 6 julio de 1764: "Trescientos Meslier distribuidos en una provincia, han conseguido muchas conversiones" (citado en Meslier 1729, p. 197). Otro hecho relevante fue la propuesta enviada a la Convención Nacional en 1793 para levantar una estatua al cura Meslier (Holbach 1822, p. 20), propuesta que fue rechazada. A pesar de la ausencia de estatuas en su honor, Meslier ha sido considerado fundador del ateísmo moderno (del anarquismo y del socialismo).

En el idioma español la obra se ha traducido como Memoria contra la religión, traducción de Javier Mina (2010), y Crítica de la religión y del Estado, antología de Menene Gras Balaguer (1978). Existe una versión más antigua titulada La religión natural. Mi testamento, editada por Casas Editoriales Maucci Hermanos e hijos, con sede en Buenos Aires y México, un ejemplar de dicha edición se encuentra en la Biblioteca Sociológica Regeneración de Ricardo Flores Magón, una colección de 87 libros que se empezaron anunciarse en su periódico en $1911 .{ }^{1}$

Debido a la enorme popularidad de la que en su momento parece que gozó la obra de Meslier, no es difícil suponer que sus ideas fueran conocidas por el autor de la Filosofía zoológica, obra que profundizaría en la explicación sobre cómo opera la naturaleza en el origen de la vida, en el origen de nuevas especies, y en origen y desarrollo de lo físico y lo moral en el ser humano.

Una influencia mucho más directa del materialismo que se encuentra en la obra de Lamarck es aquella del también materialista Pierre J. G. Cabanis, amigo de Lamarck, Holbach y Condorcet (quien, en caso del último, dio testimonio en 1781 de la presentación del escrito de Lamarck (1794) intitulado: Recherches sur les causes des principaux faits physiques). En su obra Rapports du physique et du moral de l'homme (1802), Cabanis discutió sus reflexiones materialistas respecto a la naturaleza y origen de las ideas. Como importante miembro de la Idéologie, la ciencia de las ideas, Cabanis estableció al cerebro como el órgano secretor del pensamiento (Cabanis 1815, p. xliv), un órgano cuyas ideas, determinaciones e impresiones son generadas de acuerdo con "las mismas leyes de los movimientos que [el cerebro] imprime en el órgano muscular” (Cabanis 1815, p. xlvii). En su conclusión respecto a las sensaciones y la generación de ideas, Cabanis aclara: "[e]l modo de recibir sensaciones, necesario para adquirir ideas, experimentar afectos, tener voluntades [...] [d] epende del estado de los órganos, de la fuerza o debilidad del sistema nervioso, pero particularmente del modo como [el cerebro] siente" (Cabanis 1815, p. li).

Es notorio, entonces, que Cabanis buscaba dar a conocer que la naturaleza y origen de las características emocionales y cognitivas del ser humano no poseían una naturaleza metafísica sino, al contrario, una similar a la de fenómenos fisiológicos más conocidos, como el movimiento muscular. Así mismo, para él, las ideas y sensaciones poseían un carácter material albergado en las características físicas y materiales del sistema nervioso y el cerebro. Lamarck, por su parte, compartía esta visión materialista de las ideas y las sensaciones. Su Biologie, en efecto, era un proyecto fuertemente inspirado por la Ideologie, antes mencionada, y buscaba de la misma forma una ciencia libre de preconcepciones

${ }^{1}$ Véase en http://archivomagon.net/biblioteca-digital/biblioteca-sociologica-de-regeneracion/. 
filosófico-religiosas (Corsi 2012, p. 23). Así, no es de extrañar que Cabanis fuera uno de los pocos autores que citó en sus escritos.

Como puede notarse en su obra, hay frases de Lamarck que reflejan la aceptación de la existencia de un Creador que le ha dado autonomía al mundo natural. Sin embargo, la recurrencia de citar a un Creador puede explicarse por otras razones, atribuibles al contexto en el que se publicó cada una de sus obras. En su argumentación, Lamarck sostenía que la vida se originaba por generación espontánea, la cual ocurría cada vez que factores como el calor, la humedad, la temperatura, los nutrientes, los campos magnéticos y eléctricos coincidían y hacían posible el surgimiento de formas simples. Éstas, darían origen a formas vivas que denominaba infusorios, cuyas características, en términos de Lamarck, eran "cuerpos amorfos, gelatinosos, transparentes, contráctiles y microscópicos" (Lamarck 1809, (I), p. 273). En este sentido, la Naturaleza era la causa directa del origen de la vida.

\section{Materialismo filosófico: la variación infinita}

Contrario a las interpretaciones historiográficas que sostienen o han sostenido que Lamarck era un autor deísta, en la línea de lo dicho por Pietro Corsi (2012), las ideas en las que Lamarck sustenta su visión evolutiva sobre la vida en general y la vida del ser humano son más cercanas al ateísmo o materialismo filosófico. Aunque es difícil probarlo debido a la ausencia de documentos personales y porque generalmente debemos basarnos en las obras publicadas (en las que sabemos Lamarck continuamente hace referencia a la idea de un Creador), evidencias históricas muestran que, a partir de 1803 miembros de movimientos materialistas, como la Idéologie, fueron fuertemente reprendidos por el General Bonaparte y encontraron oposición en el re-establecimiento parcial de la religión católica en Francia. Esta situación política parece haber sido la responsable del abandono del proyecto de la Biologie de parte de Lamarck, así como el inicio de un fuerte intento del naturalista de convencer a sus lectores y críticos de que había aprendido su lección y no perseguiría más líneas de investigación contrarias al estatus religioso. Lamarck sabía que, a diferencia de algunos otros intelectuales materialistas, él se encontraba en una posición política mucho más débil y se arriesgaba a una destitución total para él y su familia (Corsi 2012, p. 23).

A partir de estos momentos, el lenguaje de Lamarck se volvió más prudente y, aun cuando en sus últimas obras (de 1816 a 1820) expandiría y trabajaría su interpretación materialista de la naturaleza, se encargó de dar crédito a ideas religiosas (Corsi 2012, p. 24), principalmente al inicio e introducción de sus obras y no en los capítulos centrales donde discute sus argumentos centrales.

Por la ausencia de citas en la obra de Lamarck, no es sencillo conectar su materialismo filosófico con autores mencionados de manera directa por Lamarck, pero es obvio que conocía la obra de autores como Voltaire, Cabanis, G.L.L. Buffon, Condorcet, Denis Diderot y probablemente la obra de Meslier que se discutía en los círculos en los que se desenvolvía. Es posible pensar que puede haber una conexión directa cuando encontramos en la Filosofía zoológica de Lamarck (1809) evidencias visibles tanto de la postura nominalista con respecto al problema de la inexistencia de las especies, que había sido defendida por Buffon, a quien tampoco cita, como la idea de la variabilidad infinita, que también forma parte de acuerdo con Lamarck a las cualidades esenciales de la materia (Lamarck 1794, p. 19), y que había sido defendida por otros autores materialistas.

Sobre la variación infinita escribirá Lamarck: "[s]iempre que el hombre pretende estudiar la Naturaleza se encuentra obligado a emplear medios particulares. En primer término, para poner orden entre los objetos infinitos y variados que trata de examinar (Lamarck 1809, (I), p. 17). Continuará Lamarck:

Pero tales clasificaciones tan felizmente imaginadas por los naturalistas, así como las divisiones y subdivisiones que presenta, son medios artificiales en absoluto. Nada de todo esto, vuelvo a repetir, se encuentra en la Naturaleza, [...] De modo que se puede asegurar que entre sus producciones, la Naturaleza, no ha formado realmente ni clases, ni órdenes, ni especies constantes, sino sólo individuos que se suceden los unos a los otros y que se asemejan a los que los han producido (Lamarck 1809, (I), p. 20). 
Su idea de inexistencia de las especies o de una existencia relativa estaba firmemente ligada a la idea de la generación continua de individuos diferentes generados durante cada generación: "Se ha asignado con el nombre de especie toda colección de individuos semejantes que fueron producidos por otros individuos parecidos a ellos" (Lamarck 1809, (I), p. 20). Para Lamarck, la naturaleza con sus poderes como el de la irritabilidad está generando por un lado la vida misma y el sentimiento (Giglioni 2013, p. 37) y cada instante y en cada momento generación tras generación está generando la variación infinita de las formas orgánicas.

Esa idea de la variabilidad infinita también está asociada a la de variación infinita de las circunstancias. Cuando Lamarck señala la idea de variaciones infinitas de las circunstancias, está pensando en las múltiples posibilidades que pueden ocurrir y generar innovaciones, y aunque no utiliza el término de "contingencia", su idea de circunstancia hace referencia a la idea de una combinación entre causalidad y casualidad, que era precisamente uno de los sentidos de la idea de contingencia que se utilizaba en ese momento por las diferentes tradiciones de investigación en el contexto francés.

De estas dos ideas, la inexistencia de las especies y la variación infinita, Lamarck rompe con la idea de la fijeza o inmutabilidad de las especies y rompe con la idea de que una especie es una entidad natural [real] creada por un ser superior. Eso le permite asumir que la naturaleza orgánica está sumergida en una transformación continua: "la naturaleza sólo produce individuos que se suceden los unos a los otros y que se asemejan a los que los han producido" (Lamarck 1809, (I), p. 20).

A diferencia de los materialismos anteriores que sostuvieron la eternidad de la materia sumergida en una eterna circularidad, la concepción de Lamarck supone una materia eterna capaz de innovar:

De la magnitud y dimensión de la materia, resulta necesariamente su forma, incluyendo las variedades admirables, generadas por las modificaciones infinitas de esta materia, la belleza del universo y de alguna manera la fertilidad de la naturaleza (Lamarck 1794, p. 20).

Difícilmente Lamarck puede ser un teísta o un deísta cuando clamaba -dice Corsi (2012)- por la eternidad de la materia, y cuando establecía que la materia no podía crearse ni destruirse: "La cantidad de materia no puede aumentar o disminuir, porque ese poder que podría crear, sólo podría destruirla y ni siquiera es necesario volverla a la nada" (Lamarck 1794, p. 21).

Con sus ideas, Lamarck no sólo buscaba terminar con las creencias generalizadas sobre el mundo natural, como veremos en el siguiente apartado sus principios de transformación también eran un instrumento para explicar la naturaleza del ser humano. Lamarck consideraba que la especie humana formaba parte de la transformación general de la vida, y sus atributos como la inteligencia y la razón, eran propiedades naturales que resultaban de la organización del sistema nervioso y podían ser investigadas en términos de causas naturales (Lamarck 1809, (I), p. 187).

\section{Materialismo filosófico: razón y moral}

Lamarck imaginaba un cuadro general de la historia de la vida que comienza en el momento en que la naturaleza, por medio de las generaciones directas, espontáneas e independientes crea en forma autónoma los esbozos de la organización, que posteriormente se transformarán en infusorios o los organismos más simples. Sin embargo, esta serie de complejidad ascendente no ofrece una gradación regular, presenta diversas anomalías y separaciones, que no muestran ninguna apariencia de orden. La razón de este aparente desorden es, en opinión de Lamarck, la influencia de las circunstancias ejercidas sobre los seres vivos (Lamarck 1809, (I), p. 220). A partir de la sucesiva generación espontánea y la continua transformación de la vida, Lamarck delinea su idea la Marcha de la naturaleza (Lamarck 1809, (I), pp. 64-65).

Dentro de ese marco general, sostenía que "todos los cuerpos organizados de nuestro globo son verdaderas producciones de la Naturaleza" (Lamarck 1820, p. 65), composiciones heterogéneas que tiende a destruirse como una consecuencia inevitable del fenómeno de la vida. Bajo esa perspectiva Lamarck sugirió que en lugar de que se consideraran los clásicos tres reinos: animal, vegetal y mineral, sólo se consideran dos ramas principales los cuerpos con vida y los cuerpos sin vida (Lamarck 1830, p. 
91), reconociendo que la distancia entre los seres vivos y los elementos inorgánicos es tan grande que deben ser considerados dos cosas completamente distintas (Lamarck 1809, (I), pp. 384-385).

Como parte de los elementos de la marcha de la naturaleza, al igual que Buffon incluyó a la humanidad y rechazó su origen divino: "pensar que el hombre está dotado de un alma inmortal [...] será siempre sin bases y puramente imaginario. Si nos ocupamos de la naturaleza, ella sola debería ser el objeto de nuestros estudios" (Lamarck 1809, (II), p. 187).

El cerebro era sumamente importante para Lamarck, ya que sin esta masa principal no podría realizar el sistema nervioso ninguna de sus acciones y tampoco tendría la facultad de sentir (Lamarck 1809, (II), p. 186). De acuerdo con Lamarck, todas las funciones del sistema nervioso son provocadas por un fluido sutil,

manifiesto por los efectos que sólo este fluido podría producir, [...] remarcable por la velocidad de sus movimientos, que no podemos negar, aunque no seamos capaces de observarlo nosotros mismos, de procurarlo y someterlo a nuestras experiencias (Lamarck 1809, (II), p. 236).

Como ya se había mencionado anteriormente el materialista Meslier había considerado que el cerebro sólo era un conjunto de fibras y que nuestros pensamientos dependían de los movimientos y de las alteraciones de las fibras de nuestro cerebro (Meslier 1729, p. 87).

En su interpretación, Lamarck considera que, así como el calor o el fluido calórico es el responsable del orgasmo vital, la acción del fluido eléctrico es el que permite la acción muscular y desarrollar un sentimiento interior o de existencia que, en el caso de los organismos más "desarrollados" como los humanos, es la causa responsable de la capacidad de pensar y de emitir juicios. Para Lamarck los actos del entendimiento son: la atención, el pensamiento, la memoria y los juicios. La atención o primera de las facultades de la inteligencia, se efectúa dentro de alguna de las regiones del hipocéfalo (cerebro anterior). Es tan importante, que sin ella no se podrían formar las ideas complejas.

Para Lamarck, la segunda de las facultades de la inteligencia, el pensamiento, es un acto de reflexión sobre un objeto cualquiera, que se efectúa sobre ideas inmediatas o impresas. "La imaginación tiene su fuente en el hábito de pensar" (Lamarck 1809, (II), p. 408) esta última, una de las más bellas actividades del hombre, es la operación intelectual creadora de nuevas ideas. El genio del individuo no era otra cosa que tener una gran imaginación dirigida hacia gustos exquisitos. En este sentido, la ciencia era la disciplina que:

esencialmente sirve a todas las artes, y que no sólo nos da las mejores maneras de proveer nuestras necesidades físicas, sino que también [nos muestra] todo lo que realmente está allí, no lo que prefiriéramos que estuviera. [...] El propósito de [este arte] es la recolección de todo el conocimiento positivo que nos es posible adquirir (Lamarck 1809, (II), p. 416).

El acto formador y último de las facultades de la inteligencia es el juicio. Para Lamarck este acto "es lo más importante que el entendimiento puede ejecutar" (Lamarck 1809, (I), p. 471), y se forma como consecuencia de la relación y comparación entre ideas. Los deseos, las esperanzas, los miedos y los anhelos, nuestros razonamientos y nuestros análisis, no son más que producto de los juicios.

No es difícil aceptar el materialismo filosófico en las ideas de alguien como Lamarck que explica en términos naturales las actividades intelectuales del ser humano; que escribió que las ideas religiosas fueron creadas y divulgadas por un auto-interés de las élites para mantener el dominio sobre la población; y que afirmaba casi al final de su vida que no había principio metafísico que sustentara la vida humana y la conciencia- y que creer en la inmortalidad del alma, sólo era algo imaginario (Corsi 2012). El materialismo de Lamarck no fue ni agresivo ni antirreligioso, sino que simplemente "creyó que los sentimientos humanos y el pensamiento humano eran fenómenos naturales que podían ser explicados científicamente" (Roger en Lindberg \& Numbers 1986, p. 291), y en términos estrictamente materialistas.

En 1827 Charles Lyell, después de leer la Filosofía zoológica (Lamarck 1809) escribiría "he devorado a Lamarck [...] en un sólo viaje [...] su teoría me ha dejado encantado" (Lyell 1881, p. 168) además de 
estar encantado comprendía perfectamente las implicaciones materialistas del transformismo de Lamarck. Un par de años más tarde consideraría que, si las conjeturas de Lamarck eran correctas, entonces, terminaríamos por aceptar que toda la vida en su conjunto desciende de un sólo huevo, que estábamos emparentados con el orangután y que el ser humano tendría que renunciar a su genealogía superior (Lyell 1832, pp. 10, 14, 21-22).

\section{Conclusión}

Las ideas evolutivas de Lamarck, tienen de fondo una postura materialista tanto en el sentido metodológico como filosófico. El primer tipo de materialismo es una postura compartida por diversos autores tanto deístas como materialistas (ateísmo) que fundamentaron su ciencia en un esquema cartesiano y newtoniano. La preocupación común de todos estos autores era lograr una comprensión racional de las causas naturales de los fenómenos naturales. Lamarck utilizó su metodología materialista para abordar dos de las grandes cuestiones de la biología, el origen de la vida a partir de la materia inerte y la transformación de las especies en un ambiente dinámico.

El segundo tipo de materialismo está presente en su idea de la eternidad de la materia; en su idea de la variación infinita como un evento contingente e innovador, en una interpretación distinta a la idea de la eternidad de la materia en un sentido circular, ya que Lamarck sugiere un movimiento de la materia hacia una variación infinita, capaz de innovar; y está presente tanto en su aceptación de las capacidades intelectuales del ser humano como resultado de procesos exclusivamente naturales, como en su negación de existencia del alma.

Reconocer y entender la filosofía de la transmutación de Lamarck a través de su compromiso con el materialismo, tanto metodológico como filosófico, nos proporciona una base importante para resignificar la historia de la teoría evolutiva. Con esto, la intención primaria no es concederle a Lamarck una posición de figura única como originador de conceptos fundamentales para la comprensión de la transformación de las especies a lo largo del tiempo, sino, por un lado, destacar la necesidad de que la historia de la ciencia amplíe su enfoque a las diversas circunstancias que rodean la formación del quehacer científico. Y por el otro lado, que la comprensión de la historia de la ciencia va de la mano de discusiones filosóficas, que, entendidas en su contexto apropiado, nos abren nuevas posibilidades a la comprensión del quehacer científico, tanto antes como ahora.

\section{Bibliografía}

Cabanis, G. (1815), Rapports du physique et du moral de l'homme, Paris: Caille et Ravier.

Charbonnat, P. (2010), Historia de las filosofias materialistas, Barcelona: Biblioteca Buridán.

Coffin, V. (1917), “Censorship and Literature Under Napoleon I”, The American Historical Review 22(2): 288-308.

Corsi, P. (1987), “After the Revolution: Scientific Language and French Politics, 1795-1802”, Nuncius 2: 89-144.

Corsi, P. (2012), "Idola Tribus: Lamarck, Politics and Religion in the Early Nineteenth Century”, en Fasolo, A. (ed.), The Theory of Evolution and its Impact, Milan: Springer, pp. 11-36.

Corsi, P., Gayon, J., Gohau, G. y S. Tirard (2006), Lamarck, philosophe de la nature, Paris: Presses Universitaires de France.

Giglioni, G. (2013), “Jean-Baptiste Lamarck and the Place of Irrability in the History of Life and Death”, en Normandin, S. y C.T. Wolfe (ed.), Vitalism and the Scientific Image in Post-Enlightenment Life Science, 1800-2010, New York: Springer, pp. 19-52.

Holbach (baron d'), P.H.T. (1822), Le bon sens du curé J. Meslier, suivi de son Testament..., París: Au Palais des Thermes de Julien. 
Lamarck, J.B. (1779), Flore Françoise ou description succincte de toutes les plantes qui croissent naturellement en France, Disposée selon une nouvelle méthode d'Analyse, $\mathcal{E}$ à laquelle on a joint la citation de leurs vertus les moins équivoques en Médecine, E⿱ de leur utilité dans les Arts, Paris: Imprimerie Royale.

Lamarck, J.B. (1794), Recherches sur les causes des principaux faits physiques, Paris: Maillard.

Lamarck, J.B. (1802), Hydrogéologie ou recherches sur l'influence qu'ont les eaux sur la surface du globe terrestre; sur les causes de l'existence du bassin des mers, de son déplacement et de son transport successif sur les différens points de la surface de ce globe; enfin sur les changemens que les corps vivans exercent sur la nature et l'état de cette surface, Paris: Chez l'Auteur, Agasse et Maillard.

Lamarck, J.B. (1809), Philosophie zoologique ou exposition des considérations relatives à l'histoire naturelle des animaux, Paris: Dentu.

Lamarck, J.B. (1820), Système analytique des connaissances positives de l'homme, Paris: Chez l'Auteur et Belin.

Le Roy Ladurie, E. y G. Chaussinand-Nogaret (2010), “Athées, agnostiques et déistes au XVIIIe siècle”, Commentaire 132(4): 939-948.

Lindberg, D.C. y R.L. Numbers (eds.) (1986), God and Nature: Historical Essays on the Encounter between Christianity and Science, California: University of California Press.

Lyell, C. (1832), Principles of Geology, Being an Attempt to Explain the Former Changes of the Earth's surface, by Reference to Causes Now in Operation, Vol. 2, London: John Murray.

Lyell, C. (1881), Life, Letters, and Journals of Sir Charles Lyell, Bart, London: John Murray.

Meslier, J. (s/a [1729]), La religión natural. Mi testamento. ensayo sobre la historia natural. Jean Meslier y Juan de Antímoine, Buenos Aires-México: Editorial Maucci Hermanos é Hijos y Maucci hermanos.

Meslier, J. ([1729] 2010), Memoria contra la religión, Pamplona: Laetoli.

Meslier, J. ([1729] 1935), El buen sentido del cura Meslier, Madrid: De la Biblioteca Central de Cataluña.

Meslier, J. ([1729] 1978), Critica de la religión y del Estado, Barcelona: Ediciones Península.

Packard, A.S. (1901), Lamarck, the Founder of Evolution: His Life and Work, New York-London-Bombay: Longmans, Green and Company.

Polowetzky, M. (1993), A Bond Never Broken: The Relations Between Napoleon and the Authors of France, London: Associated University Presses.

Onfray, M. (2006), “Jean Meslier and 'The Gentle Inclination of Nature”, New Politics 10(4): 53.

Szyfman, L. (1982), Jean-Baptiste Lamarck et son époque, Paris: Masson. 